Article

\title{
Existence results of solutions for some fractional neutral functional integro-differential equations with infinite delay
}

\author{
Kazem Nouri ${ }^{1, *}$, Marjan Nazari ${ }^{1}$ and Bagher Keramati ${ }^{1}$ \\ 1 Department of Mathematics, Faculty of Mathematics, Statistics and Computer Sciences, Semnan \\ University, P. O. Box 35195-363, Semnan, Iran \\ * Correspondence: knouri@semnan.ac.ir; Tel.: +98-233-153-3204
}

\begin{abstract}
In this paper, by means of the Banach fixed point theorem and the Krasnoselskii's fixed point theorem, we investigate the existence of solutions for some fractional neutral functional integro-differential equations involving infinite delay. This paper deals with the fractional equations in the sense of Caputo fractional derivative and in the Banach spaces. Our results generalize the previous works on this issue. Also, an analytical example is presented to illustrate our results
\end{abstract}

Keywords: Fractional neutral integro-differential equations; Initial value problem; Caputo fractional derivative; Krasnoselskii's fixed point theorem

\section{Introduction}

Differential equations with fractional order appear frequently in applications as the mathematical modeling of natural phenomena in the fields of sciences and engineering including fluid flow, economics, electrical networks, and etc. (see [1-5]). In fact, most of these equations are more accurate for the description of the property of phenomena, as compared with the corresponding integer-order models. Therefore, the study of such equations has attracted a great deal of attention of researchers that we refer to the monographs such as Miller and Ross [6], Podlubny [7], Kilbas et al. [8], Diethelm [9], and some articles [10,11].

The concept of the existence theory of solutions for fractional functional differential equations with infinite delay is increasing as a necessary district of scholarships [12-17]. There are some papers dealing with this issue by using some techniques such as, fixed point theorems, the Leray-Schauder theory, method of steps, lower and upper solutions method and etc. [12,15-21]. In 2008, Benchohra et al. [19], investigated the existence of solutions for the following Riemann-Liouville fractional order functional differential equations with infinite delay using the Leray-Schauder fixed point theorem.

$$
\left\{\begin{array}{l}
D^{\alpha} x(t)=f\left(t, x_{t}\right), \text { for } t \in J=[0, T], 0<\alpha \leq 1, \\
x(t)=\phi(t), t \in(-\infty, 0]
\end{array}\right.
$$

and

$$
\left\{\begin{array}{l}
D^{\alpha}\left(x(t)-g\left(t, x_{t}\right)\right)=f\left(t, x_{t}\right), t \in J=[0, T] \\
x(t)=\phi(t), t \in(-\infty, 0] .
\end{array}\right.
$$

Also, Agarwal et al., studied the initial value problem of fractional neutral Caputo fractional derivative

$$
\left\{\begin{array}{l}
{ }^{c} D^{\alpha}\left(x(t)-g\left(t, x_{t}\right)\right)=f\left(t, x_{t}\right), \text { for } t \in J=[0, T], 0<\alpha \leq 1, \\
x_{0}=\phi \in B,
\end{array}\right.
$$

and established the existence results of solution of this problem by using Krasnoselskii's fixed point theorem [12]. Ren et al. [23], by utilizing the Banach fixed point theorem, the Leray-Schauder fixed 
point theorem and the Krasnoselskii fixed point theorem, discussed the existence and uniqueness of mild solutions in $\alpha$-norm to the following semilinear integro-differential evolution equations:

$$
\left\{\begin{array}{l}
{ }^{c} D^{\alpha} x(t)=A x(t)+f\left(t, x_{t}, \int_{0}^{t} a\left(t, s, x_{s}\right) d s\right), \text { for } t \in J=[0, T], 0<\alpha \leq 1, \\
x(t)=\phi(t), t \in(-\infty, 0],
\end{array}\right.
$$

where $A$ is the infinitesimal generator of a compact semigroup. Recently, Xie [24] and Dabas and Chauhan [25], analyzed the existence and uniqueness results for impulsive fractional integro-differential evolution equations with infinite delay, by means of Monch fixed point theorem and Kuratowski measure of noncompactness, respectively.

To close the gap, motivated and inspired by the works above, in this paper we investigate the existence of solutions for the following fractional neutral functional integral-differential equation:

$$
\left\{\begin{array}{l}
{ }^{c} D^{\alpha}\left(x(t)-g\left(t, x_{t}\right)\right)=f\left(t, x_{t}, K x(t)\right), \text { for } t \in J=[0, T], 0<\alpha \leq 1, \\
x_{0}=\phi \in B,
\end{array}\right.
$$

which is equipped with the new suitable conditions on functions $f, g$. Where ${ }^{c} D^{\alpha}$ denotes the Caputo fractional derivative, $f:[0, T] \times B \times \mathbb{R} \rightarrow \mathbb{R}$ and $g:[0, T] \times B \rightarrow \mathbb{R}$, are continuous functions, also $B$ is a phase space of mapping $(-\infty, 0]$ into $\mathbb{R}$ which will be explained in Section 2. For $x:(-\infty, T] \rightarrow \mathbb{R}$, we define $x_{t}(\theta)=x(t+\theta)$ for $t \in[0, T]$ and $-\infty<\theta \leq 0$, as well as for $k:[0, T] \times[0, T] \rightarrow[0, \infty)$, we denote

$$
K x(t)=\int_{0}^{t} k(t, s) x(s) d s
$$

with $k_{0}=\sup _{0 \leq t \leq T}\left|\int_{0}^{t} k(t, s) d s\right|$. The main tools used in this paper are Banach fixed point theorem and the Krasnoselskii's fixed point theorem.

This paper is organized as follows. In Section 2, we provide some required notation and basic concepts. In Section 3, the existence of solutions for problem (4) is analyzed under the Banach fixed point theorem and the Krasnoselskii's fixed point theorem. As a last point, an application is given in Section 4 to illustrate our theoretical results.

\section{Preliminaries}

In this section, we introduce some primary components, definitions and notations from the fractional calculus and the phase space which are used in the sequel [7].

We consider $C(J, \mathbb{R})$ as a Banach space of all continuous functions from $J$ into $\mathbb{R}$ with the norm

$$
\|x\|:=\sup _{0 \leq t \leq T}|x(t)|,
$$

where $|$.$| denotes a suitable complete norm on \mathbb{R}$. Also, $L^{1}(J, \mathbb{R})$ denotes the Banach space of measurable functions from $J$ into $\mathbb{R}$, which are Lebesgue integrable with the norm

$$
\|x\|_{L^{1}}:=\int_{J}|x(t)| d t
$$

The fractional integral of order $\alpha>0$ of a function $x \in L^{1}(J, \mathbb{R})$ is defined as

$$
I^{\alpha} x(t)=\frac{1}{\Gamma(\alpha)} \int_{0}^{t}(t-s)^{\alpha-1} x(s) d s,
$$

where $\Gamma($.$) is the Gamma function.$

Let $n-1<\alpha \leq n$, the $\alpha$-th Caputo derivative of $x \in C(J, \mathbb{R})$ is defined as

$$
{ }^{c} D^{\alpha} x(t)=\frac{1}{\Gamma(n-\alpha)} \int_{0}^{t}(t-s)^{n-\alpha-1} x^{(n)}(s) d s .
$$


In this paper, to describe fractional neutral functional integro-differential equations with infinite delay, we assume an evident definition of the phase space $\left(B,\|\cdot\|_{B}\right)$ such that is a seminormed linear space of functions mapping $(-\infty, 0]$ into $\mathbb{R}$ and satisfies in the following fundamental axioms $[19,26]$ : $(A)$ : for every $x:(-\infty, T] \rightarrow \mathbb{R}$ with $x_{0} \in B$ and $t \in[0, T]$, the following conditions hold:

$$
\begin{aligned}
& \text { (i) } x_{t} \in B \\
& \text { (ii) }\left\|x_{t}\right\|_{B} \leq N(t) \sup _{0 \leq s \leq t}|x(s)|+M(t)\left\|x_{0}\right\|_{B} \\
& \text { (iii) }|x(t)| \leq h\left\|x_{t}\right\|_{B},
\end{aligned}
$$

where $h \geq 0$ is a constant, $N \in C([0, T],[0, \infty)), M:[0, T] \rightarrow[0, \infty)$ is locally bounded and $h, N$ and $M$ are independent of $x($.$) .$

$(A-1)$ : For $x($.$) satisfies in (A), x_{t} \in C([0, T], B)$.

$(A-2)$ : The space $B$ is complete.

Furthermore, the following notations are used in this paper,

$$
n_{T}=\sup _{0 \leq t \leq T}|N(t)|, \quad m_{T}=\sup _{0 \leq t \leq T}|M(t)|
$$

and

$$
\Omega=\left\{x:(-\infty, T] \rightarrow \mathbb{R},\left.x\right|_{(-\infty, 0]} \in B,\left.\quad x\right|_{[0, T]} \text { is continuous }\right\} .
$$

\section{Main result}

In this section, we study the existence of solutions Eq. (4), to demonstrate our results. we list the following assumptions:

$\left(H_{1}\right)$ : Let $f: J \times B \times \mathbb{R} \rightarrow \mathbb{R}$ be a continuous function and for each $\left(t, x_{t}, K x(t)\right),\left(t, y_{t}, K y(t)\right) \in$ $J \times B \times \mathbb{R}$, there exist $L_{i}(t) \in C([0, T],[0, \infty)), i=1,2$, such that

$$
\left\|f\left(t, x_{t}, K x(t)\right)-f\left(t, y_{t}, K y(t)\right)\right\| \leq L_{1}(t)\left\|x_{t}-y_{t}\right\|_{B}+L_{2}(t)\|K x-K y\| .
$$

$\left(H_{2}\right)$ : Let $g: J \times B \rightarrow \mathbb{R}$ be a continuous function and for each $\left(t, x_{t}\right),\left(t, y_{t}\right) \in J \times B$, there exist $L_{3}(t) \in C([0, T],[0, \infty))$, such that

$$
\left\|g\left(t, x_{t}\right)-g\left(t, y_{t}\right)\right\| \leq L_{3}(t)\left\|x_{t}-y_{t}\right\|_{B}
$$

also, for $\left\|L_{3}(t)\right\|=\gamma_{1}$, we assume $\gamma_{1} n_{T}<1$.

$\left(H_{3}\right)$ : The constants $I_{L}^{\alpha}>0$ and $\lambda_{1}<1$ are determined by

$$
\begin{gathered}
I_{L}^{\alpha}=\max \left\{\sup _{0 \leq t \leq T}\left|I^{\alpha} L_{i}(t)\right|, i=1,2\right\}, \\
\lambda_{1}=\left[\gamma_{1} n_{T}+\left(k_{0}+n_{T}\right) I_{L}^{\alpha}\right] .
\end{gathered}
$$

A function $x \in \Omega$ is a solution of problem (4) with initial condition $x_{0}=\phi \in B$, if $x$ satisfies in the following integral equation

$$
\left\{\begin{aligned}
x(t)= & \phi(0)-g(0, \phi)+g\left(t, x_{t}\right) \\
& +\frac{1}{\Gamma(\alpha)} \int_{0}^{t}(t-s)^{\alpha-1} f\left(s, x_{s}, K x(s)\right) d s, \quad t \in[0, T], \\
x_{0}=\phi . &
\end{aligned}\right.
$$

Proof. Assume that $x$ is a solution of (4), therefore, for each $t \in J$, we have 


$$
{ }^{c} D^{\alpha}\left(x(t)-g\left(t, x_{t}\right)\right)=f\left(t, x_{t}, K x(t)\right) .
$$

Applying the Riemann-Liouville fractional integral operator on both sides, we obtain

$$
x(t)-g\left(t, x_{t}\right)+c_{1}=\frac{1}{\Gamma(\alpha)} \int_{0}^{t}(t-s)^{\alpha-1} f\left(s, x_{s}, K x(s)\right) d s .
$$

Using the initial condition, we get

$$
c_{1}=-\phi(0)+g(0, \phi)
$$

Thus

$$
x(t)=\phi(0)-g(0, \phi)+g\left(t, x_{t}\right)+\frac{1}{\Gamma(\alpha)} \int_{0}^{t}(t-s)^{\alpha-1} f\left(s, x_{s}, K x(s)\right) d s .
$$

and $x$ is a solution of the integral equation (6).

Assume that the hypotheses $\left(H_{1}\right)-\left(H_{3}\right)$ are satisfied. Therefore, the problem (4) has a unique solution.

Proof. Firstly, in order to prove this theorem, we need to transform problem (4) into a fixed point problem. Therefore, from the Lemma 1, consider the operator $\Lambda: \Omega \rightarrow \Omega$ defined as

$$
\Lambda x(t)=\left\{\begin{array}{l}
\phi(0)-g(0, \phi)+g\left(t, x_{t}\right) \\
\quad+\frac{1}{\Gamma(\alpha)} \int_{0}^{t}(t-s)^{\alpha-1} f\left(s, x_{s}, K x(s)\right) d s, \quad t \in[0, T] \\
\phi(t), \quad t \in(-\infty, 0] .
\end{array}\right.
$$

Also, we define $\varphi():.(-\infty, T] \rightarrow \mathbb{R}$ by

$$
\varphi(t)= \begin{cases}\phi(0), & t \in[0, T] \\ \phi(t), & t \in(-\infty, 0]\end{cases}
$$

thus $\varphi(t) \in \Omega$ and $\varphi_{0}=\phi$. Let $x(t)=y(t)+\varphi(t)$, which implies $x_{t}=y_{t}+\varphi_{t}$, for each $t \in[0, T]$. It is evident that $x$ satisfies in Eq. (6) if and only if $y_{0}=0$ and also, the function $y($.$) satisfies in the$ following equation,

$$
y(t)=-g(0, \phi)+g\left(t, y_{t}+\varphi_{t}\right)+\frac{1}{\Gamma(\alpha)} \int_{0}^{t}(t-s)^{\alpha-1} f\left(s, y_{s}+\varphi_{s}, K(y+\varphi)(s)\right) d s
$$

Set

$$
\widetilde{B}=\left\{y \in \Omega: y_{0}=0\right\},
$$

and let $\|\cdot\|_{\widetilde{B}}$ be the seminorm in $\widetilde{B}$ defined by

$$
\|y\|_{\widetilde{B}}=\left\|y_{0}\right\|_{B}+\sup _{0 \leq t \leq T}|y(t)|=\|y\|,
$$

thus, $\left(\widetilde{B},\|\cdot\|_{\widetilde{B}}\right)$ is a Banach space. We define the operator $\widetilde{\Lambda}: \widetilde{B} \rightarrow \widetilde{B}$ as follows

$$
\widetilde{\Lambda} y(t)=\left\{\begin{array}{l}
-g(0, \phi)+g\left(t, y_{t}+\varphi_{t}\right) \\
\quad+\frac{1}{\Gamma(\alpha)} \int_{0}^{t}(t-s)^{\alpha-1} f\left(s, y_{s}+\varphi_{s}, K(y+\varphi)(s)\right) d s, \quad t \in[0, T] \\
0, \quad t \in(-\infty, 0] .
\end{array}\right.
$$


It is clear that the operator $\widetilde{\Lambda}$ has a fixed point if and only if $\Lambda$ has a fixed point. So, our aim is to show that the operator $\widetilde{\Lambda}$ has a fixed point.

From the assumption $(A-i i)$, we get the following estimate,

$$
\begin{aligned}
\left\|\varphi_{t}\right\|_{B} & \leq N(t) \sup _{0 \leq s \leq t}|\varphi(s)|+M(t)\left\|\varphi_{0}\right\|_{B} \\
& \leq n_{T}|\phi(0)|+m_{T}\|\phi\|_{B}=\eta .
\end{aligned}
$$

On the other hand, since the functions $f, g$ are continuous and $\left\|\varphi_{t}\right\|_{B} \leq \eta$, therefore, there exist the constants $\gamma_{2}, \gamma_{3}$, such that

$$
\gamma_{2}=\left\|f\left(s, \varphi_{s}, K \varphi(s)\right)\right\|, \gamma_{3}=\left\|g\left(s, \varphi_{s}\right)\right\|
$$

Choosing

$$
R_{1} \geq \frac{\gamma_{2} T^{\alpha}}{\Gamma(\alpha+1)}+\gamma_{3}+\|g(0, \phi)\|+\left[\gamma_{1} n_{T}+\left(k_{0}+n_{T}\right) I_{L}^{\alpha}\right] R_{1}
$$

and considering the set $D_{R_{1}}=\left\{y \in \widetilde{B}:\|y\| \leq R_{1}\right\}$, clearly $D_{R_{1}}$ is a closed, bounded and convex set of $\widetilde{B}$.

For every $y \in D_{R_{1}}$, by means of $\left(H_{1}\right),\left(H_{2}\right),(A-i i)$ and triangle inequality, we get

$$
\begin{aligned}
&\left\|f\left(s, y_{s}+\varphi_{s}, K(y+\varphi)(s)\right)\right\| \\
& \leq\left\|f\left(s, y_{s}+\varphi_{s}, K(y+\varphi)(s)\right)-f\left(s, \varphi_{s}, K \varphi(s)\right)\right\| \\
&+\left\|f\left(s, \varphi_{s}, K \varphi(s)\right)\right\| \\
& \leq L_{1}(s)\left\|y_{s}\right\|_{B}+L_{2}(s)\|K y\|+\gamma_{2} \\
& \leq L_{1}(s) n_{T} \sup _{0 \leq \eta \leq s}|y(\eta)|+L_{2}(s) k_{0}\|y\|+\gamma_{2} \\
& \leq {\left[n_{T} L_{1}(s)+k_{0} L_{2}(s)\right] R_{1}+\gamma_{2}, }
\end{aligned}
$$

and

$$
\begin{aligned}
\left\|g\left(s, y_{s}+\varphi_{s}\right)\right\| & \leq\left\|g\left(s, y_{s}+\varphi_{s}\right)-g\left(s, \varphi_{s}\right)\right\|+\left\|g\left(s, \varphi_{s}\right)\right\| \\
& \leq L_{3}(s)\left\|y_{s}\right\|_{B}+\gamma_{3} \leq \gamma_{1} n_{T} R_{1}+\gamma_{3} .
\end{aligned}
$$

Now, we show that $\widetilde{\Lambda}\left(D_{R_{1}}\right) \subseteq D_{R_{1}}$. Recalling $\left(H_{3}\right)$, (9) and (10), we get

$$
\begin{aligned}
\|\widetilde{\Lambda} y\| \leq & \gamma_{1} n_{T} R_{1}+\gamma_{3}+\|g(0, \phi)\| \\
& +\left\|\frac{1}{\Gamma(\alpha)} \int_{0}^{t}(t-s)^{\alpha-1}\left(\left[n_{T} L_{1}(s)+k_{0} L_{2}(s)\right] R_{1}+\gamma_{2}\right) d s\right\| \\
\leq & \frac{\gamma_{2} T^{\alpha}}{\Gamma(\alpha+1)}+\gamma_{3}+\|g(0, \phi)\|+\left[\gamma_{1} n_{T}+\left(k_{0}+n_{T}\right) I_{L}^{\alpha}\right] R_{1} \leq R_{1},
\end{aligned}
$$

thus, $\|\widetilde{\Lambda} y\|_{\widetilde{B}} \leq R_{1}$.

Next, we shall show that $\widetilde{\Lambda}$ is a contraction mspping. For $u(t), v(t) \in D_{R_{1}}$ and $t \in[0, T]$, we obtain 


$$
\begin{aligned}
&\|\widetilde{\Lambda} u-\widetilde{\Lambda} v\| \\
& \leq \quad\left\|g\left(t, u_{t}+\varphi_{t}\right)-g\left(t, v_{t}+\varphi_{t}\right)\right\|+\| \frac{1}{\Gamma(\alpha)} \int_{0}^{t}(t-s)^{\alpha-1} \\
&\left\|f\left(s, u_{s}+\varphi_{s}, K(u+\varphi)(s)\right)-f\left(s, v_{s}+\varphi_{s}, K(v+\varphi)(s)\right)\right\| d s \| \\
& \leq L_{3}(t)\left\|u_{t}-v_{t}\right\|_{B}+\| \frac{1}{\Gamma(\alpha)} \int_{0}^{t}(t-s)^{\alpha-1} \\
& {\left[L_{1}(s)\left\|u_{s}-v_{s}\right\|_{B}+L_{2}(s)\|K u-K v\|\right] d s \| } \\
& \leq \gamma_{1} n_{T}\|u-v\|+\| \frac{1}{\Gamma(\alpha)} \int_{0}^{t}(t-s)^{\alpha-1}\left[L_{1}(s) n_{T} \sup _{0 \leq \eta \leq s}|u(\eta)-v(\eta)|\right. \\
&\left.+L_{2}(s) k_{0}\|u-v\|\right] d s \| \\
& \leq {\left[\gamma_{1} n_{T}+\left(k_{0}+n_{T}\right) I_{L}^{\alpha}\right]\|u-v\|=\lambda_{1}\|u-v\|_{\widetilde{B}} . }
\end{aligned}
$$

Therefore,

$$
\|\widetilde{\Lambda} u-\widetilde{\Lambda} v\|_{\widetilde{B}} \leq \lambda_{1}\|u-v\|_{\widetilde{B}}
$$

where $\lambda_{1}$ is given in $\left(H_{3}\right)$. Finally, we deduce $\Lambda$ has a unique fixed point by means of the contraction mapping principle.

Utilize of fixed point theorems is a suitable tool for proving the existence and uniqueness of different equations (for instance see $[15,16,27,28]$ and the references therein). For this purpose, in the following we will use of the Krasnoselskii fixed point theorem.

(Krasnoselskii's Fixed Point Theorem [12,29]). Let $X$ be a Banach space, $E$ be a bounded closed convex subset of $X$, and let $S, U$ be maps of $E$ into $X$ such that $S x+U y \in E$ for every pair $x, y \in E$. If $S$ is a contraction and $U$ is completely continuous, then the equation

$$
S x+U x=x
$$

has at least one solution on $E$.

Now, we present the subsequent assumptions:

$\left(H_{4}\right)$ : Let $f: J \times B \times \mathbb{R} \rightarrow \mathbb{R}$ be the continuous function and for each $\left(t, x_{t}, K x(t)\right) \in J \times B \times \mathbb{R}$, there exist $P_{i}(t) \in C([0, T],[0, \infty)), i=1,2$, such that,

$$
\| f\left(t, x_{t}, K x(t)\left\|\leq P_{1}(t)\right\| x_{t}\left\|_{B}+P_{2}(t)\right\| K x(t) \| .\right.
$$

$\left(H_{5}\right)$ : The constant $I_{P}^{\alpha}>0$ is determined by

$$
I_{P}^{\alpha}=\max \left\{\sup _{0 \leq t \leq T}\left|I^{\alpha} P_{i}(t)\right|, i=1,2\right\} .
$$

Assume that the hypotheses $\left(H_{2}\right)$ and $\left(H_{4}\right)-\left(H_{5}\right)$ are satisfied. Then, the problem (4) has at least one solution.

Proof. Choosing 


$$
\begin{aligned}
R_{2} \geq & \gamma_{1} n_{T} R_{2}+\gamma_{3}+\|g(0, \phi)\| \\
& +\left[n_{T} R_{2}+m_{T}\|\phi\|_{B}+n_{T}|\phi(0)|+k_{0}\left(R_{2}+|\phi(0)|\right)\right] I_{P}^{\alpha}
\end{aligned}
$$

We define the set $D_{R_{2}}=\left\{y \in \widetilde{B}:\|y\|_{\widetilde{B}} \leq R_{2}\right\}$, so $D_{R_{2}}$ is a closed, bounded and convex set of Banach space $\widetilde{B}$. Also, we consider operators $\widetilde{\Lambda}_{1}$ and $\widetilde{\Lambda}_{2}$ on $D_{R_{2}}$ as

$$
\begin{aligned}
& \widetilde{\Lambda}_{1} y(t)=\left\{\begin{array}{l}
-g(0, \phi)+g\left(t, y_{t}+\varphi_{t}\right), \quad t \in[0, T] \\
0, \quad t \in(-\infty, 0],
\end{array}\right. \\
& \widetilde{\Lambda}_{2} y(t)=\left\{\begin{array}{l}
\frac{1}{\Gamma(\alpha)} \int_{0}^{t}(t-s)^{\alpha-1} f\left(s, y_{s}+\varphi_{s}, K(y+\varphi)(s)\right) d s, \quad t \in[0, T], \\
0, \quad t \in(-\infty, 0] .
\end{array}\right.
\end{aligned}
$$

Next, we are going to show that $\widetilde{\Lambda}_{1}+\widetilde{\Lambda}_{2}$ has a fixed point in $D_{R_{2}}$. Since the proof of the theorem is long, we split it into several steps.

Step 1. $\widetilde{\Lambda}\left(D_{R_{2}}\right) \subset D_{R_{2}}$ for some $R_{2}>0$.

Let $u(t), v(t) \in D_{R_{2}}$, by $\left(H_{2}\right),\left(H_{4}\right)$ and $\left(H_{5}\right)$, we obtain

$$
\begin{aligned}
\left\|\widetilde{\Lambda}_{1} u+\tilde{\Lambda}_{2} v\right\| \leq & \|g(0, \phi)\|+\left\|g\left(t, \varphi_{t}\right)\right\|+\left\|g\left(t, u_{t}+\varphi_{t}\right)-g\left(t, \varphi_{t}\right)\right\| \\
& +\frac{1}{\Gamma(\alpha)}\left\|\int_{0}^{t}(t-s)^{\alpha-1} f\left(s, v_{s}+\varphi_{s}, K(v+\varphi)(s)\right) d s\right\| \\
\leq & \gamma_{1} n_{T} R_{2}+\gamma_{3}+\|g(0, \phi)\|+\frac{1}{\Gamma(\alpha)} \| \int_{0}^{t}(t-s)^{\alpha-1} \\
& {\left[P_{1}(s)\left\|v_{s}+\varphi_{s}\right\|_{B}+P_{2}(s)\|K(v+\varphi)\|\right] d s \| } \\
\leq & \gamma_{1} n_{T} R_{2}+\gamma_{3}+\|g(0, \phi)\| \\
& +\left[n_{T} R_{2}+m_{T}\|\phi\|_{B}+n_{T}|\phi(0)|+k_{0}\left(R_{2}+|\phi(0)|\right)\right] I_{P}^{\alpha} \leq R_{2},
\end{aligned}
$$

since

$$
\left\|v_{s}+\varphi_{s}\right\|_{B} \leq\left\|v_{s}\right\|_{B}+\left\|\varphi_{s}\right\|_{B} \leq n_{T} R_{2}+m_{T}\|\phi\|_{B}+n_{T}|\phi(0)|=\eta^{*},
$$

and

$$
\|K(v+\varphi)\| \leq k_{0}\|v+\varphi\| \leq k_{0}\left(R_{2}+|\phi(0)|\right)=\eta^{* *},
$$

thus, $\left\|\widetilde{\Lambda}_{1} u+\widetilde{\Lambda}_{2} v\right\|_{\widetilde{B}} \leq R_{2}$.

Step 2. $\widetilde{\Lambda}_{1}$ is a contraction on $D_{R_{2}}$.

For every $u(t), v(t) \in D_{R_{2}}$ and $t \in[0, T]$, by means of $\left(H_{2}\right)$, we get

$$
\begin{aligned}
\left\|\widetilde{\Lambda}_{1} u-\widetilde{\Lambda}_{1} v\right\|_{\widetilde{B}} & =\left\|\widetilde{\Lambda}_{1} u-\widetilde{\Lambda}_{1} v\right\|=\left\|g\left(t, u_{t}+\varphi_{t}\right)-g\left(t, v_{t}+\varphi_{t}\right)\right\| \\
& \leq L_{3}(t)\left\|u_{t}-v_{t}\right\|_{B} \leq \gamma_{1} n_{T}\|u-v\|=\gamma_{1} n_{T}\|u-v\|_{\widetilde{B}},
\end{aligned}
$$

in view of $0<\gamma_{1} n_{T}<1, \widetilde{\Lambda}_{1}$ is a contraction on $D_{R_{2}}$.

Step 3. $\widetilde{\Lambda}_{2}$ is a completely continuous operator.

The continuity of $f$ implies that the operator $\widetilde{\Lambda}_{2}$ is continuous, we show that $\widetilde{\Lambda}_{2}$ is uniformly bounded on $D_{R_{2}}$. Since 


$$
\begin{aligned}
\left\|\widetilde{\Lambda}_{2} v\right\|_{\widetilde{B}}=\left\|\widetilde{\Lambda}_{2} v\right\| & =\left\|\frac{1}{\Gamma(\alpha)} \int_{0}^{t}(t-s)^{\alpha-1} f\left(s, v_{s}+\varphi_{s}, K(v+\varphi)(s)\right) d s\right\| \\
& \leq\left(\eta^{*}+\eta^{* *}\right) I_{P}^{\alpha},
\end{aligned}
$$

hence, $\left\{\widetilde{\Lambda}_{2} v(t): v(t) \in D_{R_{2}}\right\}$ is uniformly bounded. obtain

Finally, we prove that $\left\{\widetilde{\Lambda}_{2} v(t): v(t) \in D_{R_{2}}\right\}$ is equicontinuous. For every $0 \leq t_{1} \leq t_{2} \leq T$, we

$$
\begin{aligned}
\left|\widetilde{\Lambda}_{2} v\left(t_{2}\right)-\widetilde{\Lambda}_{2} v\left(t_{1}\right)\right| & \\
\leq & \frac{1}{\Gamma(\alpha)} \int_{0}^{t_{1}}\left[\left(t_{2}-s\right)^{\alpha-1}-\left(t_{1}-s\right)^{\alpha-1}\right]\left|f\left(s, v_{s}+\varphi_{s}, K(v+\varphi)(s)\right)\right| d s \\
& +\frac{1}{\Gamma(\alpha)} \int_{t_{1}}^{t_{2}}\left(t_{2}-s\right)^{\alpha-1}\left|f\left(s, v_{s}+\varphi_{s}, K(v+\varphi)(s)\right)\right| d s \\
\leq & \frac{1}{\Gamma(\alpha)} \int_{0}^{t_{1}}\left[\left(t_{2}-s\right)^{\alpha-1}-\left(t_{1}-s\right)^{\alpha-1}\right]\left[P_{1}(s)\left\|v_{s}+\varphi_{s}\right\|_{B}\right. \\
& \left.+P_{2}(s)\|K(v+\varphi)\|\right] d s+\frac{1}{\Gamma(\alpha)} \int_{t_{1}}^{t_{2}}\left(t_{2}-s\right)^{\alpha-1}\left[P_{1}(s)\left\|v_{s}+\varphi_{s}\right\|_{B}\right. \\
& \left.+P_{2}(s)\|K(v+\varphi)\|\right] d s \\
\leq & \frac{\left(\left\|P_{1}\right\| \eta^{*}+\left\|P_{2}\right\|\right) \eta^{* *}}{\Gamma(\alpha)}\left[\int_{0}^{t_{1}}\left[\left(t_{2}-s\right)^{\alpha-1}-\left(t_{1}-s\right)^{\alpha-1}\right] d s\right. \\
& \left.+\int_{t_{1}}^{t_{2}}\left(t_{2}-s\right)^{\alpha-1} d s\right] \\
= & \frac{\left(\left\|P_{1}\right\| \eta^{*}+\left\|P_{2}\right\|\right) \eta^{* *}}{\Gamma(\alpha+1)}\left[\left(t_{2}-t_{1}\right)^{\alpha}+t_{1}^{\alpha}-t_{2}^{\alpha}+\left(t_{2}-t_{1}\right)^{\alpha}\right] \\
\leq & \frac{2\left(\left\|P_{1}\right\| \eta^{*}+\left\|P_{2}\right\|\right) \eta^{* *}}{\Gamma(\alpha+1)}\left(t_{2}-t_{1}\right)^{\alpha} .
\end{aligned}
$$

As $t_{1} \rightarrow t_{2}$ the right-hand side of the above inequality tends to zero. It means that $\left\{\widetilde{\Lambda}_{2} v(t): v(t) \in D_{R_{2}}\right\}$ is equicontinuous. Also, the results of steps 1-3, together with the Arzela-Ascoli theorem imply that $\widetilde{\Lambda}_{2}$ is a completely continuous operator. The conclusion of the theorem holds by using the Krasnoselskii's fixed point theorem.

\section{Application}

To illustrate the application of the obtained results, we consider the following example,

$$
\begin{aligned}
& { }^{c} D^{\frac{1}{2}}\left[x(t)-\frac{1}{8} \int_{-\infty}^{t} e^{2 s-t} x(s) d s\right]=\frac{1}{8} \int_{-\infty}^{t} t e^{2(s-t)} x(s) d s+\frac{1}{8} \int_{0}^{t} \sin (t-s) x(s) d s, \quad t \in[0,1] \\
& x(t)=1, \quad t \in(-\infty, 0]
\end{aligned}
$$

Let 


$$
\begin{aligned}
& B=\left\{\phi:(-\infty, 0] \rightarrow \mathbb{R}, \int_{-\infty}^{0} h(s)\|\phi\|_{[s, 0]} d s<\infty\right\} \\
& \|\phi\|_{B}=\int_{-\infty}^{0} h(s)\|\phi\|_{[s, 0]} d s
\end{aligned}
$$

where $\|\phi\|_{[s, 0]}=\sup _{t \in[s, 0]}|\phi(t)|$ and $h:(-\infty, 0] \rightarrow(0, \infty)$ is a continuous function with $l=$ $\int_{-\infty}^{0} h(s) d s<\infty$. For phase space, we choose $h(s)=e^{2 s}, s<0$, then $l=\frac{1}{2}$. Also, we give

$$
\begin{aligned}
& g(t, \phi)=\frac{e^{t}}{8} \int_{-\infty}^{0} e^{2 s} \phi(s) d s, \\
& f(t, \phi, K x(t))=\frac{t}{8} \int_{-\infty}^{0} e^{2 s} \phi(s) d s+K x(t), \\
& K x(t)=\frac{1}{8} \int_{0}^{t} \sin (t-s) x(s) d s .
\end{aligned}
$$

Hence, the equation (11) can be written in the abstract form of the equation (4). Now, for $t \in$ $[0,1], \phi_{1}, \phi_{2} \in B$, and $x_{1}, x_{2} \in C([0,1], \mathbb{R})$, we obtain

$$
\begin{aligned}
\left|f\left(t, \phi_{1}, K x_{1}(t)\right)-f\left(t, \phi_{2}, K x_{2}(t)\right)\right| \leq & \left|\frac{t}{8} \int_{-\infty}^{0} e^{2 s}\left(\phi_{1}(s)-\phi_{2}(s)\right) d s\right|+\mid \frac{1}{8} \int_{0}^{t} \sin (t-s) \\
& \times\left(x_{1}(s)-x_{2}(s)\right) d s \mid \\
\leq & \frac{t}{8} \int_{-\infty}^{0} e^{2 s}\left|\phi_{1}(s)-\phi_{2}(s)\right| d s \\
& +\left|\frac{1}{8} \int_{0}^{t} \sin (t-s)\left(x_{1}(s)-x_{2}(s)\right) d s\right| \\
\leq & \frac{t}{8} \int_{-\infty}^{0} e^{2 s}\left\|\phi_{1}(s)-\phi_{2}(s)\right\|_{[s, 0]} d s+\frac{1}{8}\left|K x_{1}(t)-K x_{2}(t)\right| \\
= & L_{1}(t)\left\|\phi_{1}-\phi_{2}\right\|_{B}+L_{2}(t)\left|K x_{1}(t)-K x_{2}(t)\right|,
\end{aligned}
$$

and

$$
\begin{aligned}
\left|g\left(t, \phi_{1}\right)-g\left(t, \phi_{2}\right)\right| & \leq\left|\frac{e^{t}}{8} \int_{-\infty}^{0} e^{2 s}\left(\phi_{1}(s)-\phi_{2}(s)\right) d s\right| \leq \frac{e^{t}}{8} \int_{-\infty}^{0} e^{2 s}\left|\phi_{1}(s)-\phi_{2}(s)\right| d s \\
& \leq \frac{e^{t}}{8} \int_{-\infty}^{0} e^{2 s}\left\|\phi_{1}(s)-\phi_{2}(s)\right\|_{[s, 0]} d s=L_{3}(t)\left\|\phi_{1}-\phi_{2}\right\|_{B^{\prime}}
\end{aligned}
$$

and

$$
\begin{aligned}
\left|f\left(t, \phi_{1}, K x_{1}(t)\right)\right| & \leq\left|\frac{t}{8} \int_{-\infty}^{0} e^{2 s} \phi_{1}(s) d s\right|+\left|\frac{1}{8} \int_{0}^{t} \sin (t-s) x_{1}(s) d s\right| \\
& \leq P_{1}(t)\left\|\phi_{1}\right\|_{B}+P_{2}(t)\left|K x_{1}(t)\right|
\end{aligned}
$$

where $L_{1}(t)=P_{1}(t)=\frac{t}{8}, L_{2}(t)=P_{2}(t)=\frac{1}{8}, L_{3}(t)=\frac{e^{t}}{8}$. Furthermore, we get that $n_{T}=\frac{1}{2}, \gamma_{1}=$ $\frac{e}{8}, k_{0}=1, I_{L}^{\frac{1}{2}}=\frac{1}{8 \Gamma\left(\frac{3}{2}\right)}$ and

$$
\lambda_{1}=\left[\gamma_{1} n_{T}+\left(k_{0}+n_{T}\right) I_{L}^{\frac{1}{2}}\right]<1 .
$$


Thus the conditions $\left(H_{1}\right)-\left(H_{5}\right)$ are fulfilled. We realize that the equation (11) has a unique solution on $[0,1]$.

\section{Conclusion}

In this paper, we discussed the existence results for a class of fractional neutral functional integro-differential equations with time-dependent delay. Using the Banach fixed point theorem and the Krasnoselskii's fixed point theorem some results are presented. The new conditions are assumed in our works which we can generalize for another problems in the future. To validate the obtained theoretical results, we analyzed one example.

\section{Bibliography}

1. Bassukas, I.D. Comparative Gompertzian analysis of alterations of tumor growth patterns. Cancer. Res. 1994, 54, 4385-4392.

2. Bassukas, I.D.; Schultze, B.M. The recursion formula of the Gompertz function: a simple method for the estimation and comparison of tumor growth curves. Growth. Develop. Aging. 1998, 52, 113-122.

3. Doha, E.H.; Bhrawy, A.H.; Baleanu, D.; Ezz-Eldien, S.S.; Hafez, R.M. An efficient numerical scheme based on the shifted orthonormal Jacobi polynomials for solving fractional optimal control problems. Advan. Differ. Equat. 2015, doi: 10.1186/s13662-014- 0344-z.

4. Doha, E.H.; Bhrawy, A.H.; Ezz-Eldien, S.S. An efficient Legendre spectral tau matrix formulation for solving fractional subdiffusion and reaction subdiffusion equations. Comput. Nonl. Dyn. 2015, 10, doi: $10.1115 / 1.4027944$.

5. Jafari, H.; Khaliquea, C.M.; Nazari, M. An algorithm for the numerical solution of nonlinear fractional-order Van der Pol oscillator equation. Math. Comput. Model. 2012, 55, 1782-1786.

6. Miller, K.S.; Ross, B. An Introduction to the Fractional Calculus and Fractional Differential Equations. Wiley: New york, 1993.

7. Podlubny, I. Fractional Differential Equations. Academic Press: San Diego, 1999.

8. Killbas, A.A.; Srivastava, H.M.; Trujillo, J.J. Theory and Applications of Fractional Differential Equations. Elsevier: Amesterdam, 2006.

9. Diethelm, K. The Analysis of Differential Equations. Springer-Verlag: Berlin, 2010.

10. Diethelm, K. Analysis of fractional differential equations. Math. Anal. Appl. 2002, 265, 229-248.

11. Henderson, J.; Ouahab, A. Fractional functional differential inclusions with finite delay. Nonlinear. Anal. 2009, 70, 2091-2105.

12. Agarwal, R.P.; Zhou, Y.; He, Y. Existence of fractional neutral functional differential equations. Comput. Math. Appl. 2010, 59, 1095-1100.

13. Alvan, M.; Darzi, R.; Mahmoodi, A. Existence Results for a New Class of Boundary Value Problems of Nonlinear Fractional Differential Equations. Mdpi. 2016, doi: 10.3390/math4010013.

14. Suganya, S.; Mallika Arjunan, M.; Existence of Mild Solutions for Impulsive Fractional Integro-Differential Inclusions with State-Dependent Delay. Mdpi. 2017, doi: 10.3390/math5010009.

15. Chadha, A.; Pandey, D.N. Existence and approximation of solution to neutral fractional differential equation with nonlocal conditions. Comput. Math. Appl. 2015, 69, 893-908.

16. Chadha, A.; Pandey, D.N. Existence results for an impulsive neutral stochastic fractional integro-differential equation with infinite delay. Nonlinear. Anal. 2015, 128, 149-175.

17. Ravichandran, C.; Baleanu, D. Existence results for fractional neutral functional integro-differential evolution equations with infinite delay in Banach spaces. Adv. Differ. Equ. 2013, 215, 142-149.

18. Balachandran, K.; Kiruthika, S.; Trujillo, J.J. Existence results for fractional impulsive integro-differential equations in Banach spaces. Commun. Nonlinear. Sci. Numer. Simul. 2011, 16, 1970-1977.

19. Benchohra, M.; Henderson, J.; Ntouyas, S.K.; Ouahab, A. Existence results for fractional order functional differential equations with infinite delay. Math. Anal. Appl. 2008, 338, 1340-1350.

20. Kaslik, E.; Sivasundaram, S. Analytical and numerical methods for the stability analysis of linear fractional delay differential equations. Comput. Appl. Math. 2012, 236, 4027-4041. 
21. Suganya, S.; Baleanu, D.; Kalamani, P.; Arjunan, M.M. On fractional neutral integro-differential systems with state-dependent delay and non-instantaneous impulses. Adv. Differ. Equ. 2015, 372, 142-149.

22. Lakshmikantham, V. Theory of fractional functional differential equations. Nonlinear. Anal. 2008, 69, 3337-3343.

23. Ren, Y.; Qin, Y.; Sakthivel, R. Existence results for fractional order semilinear integro-differential evolution equations with infinite delay. Integr. Equ. Oper. Theory. 2010, 67, 33-49.

24. Xie, S. Existence results of mild solutions for impulsive fractional integro-differential evolution equations with infinite delay. Fract. Calc. Appl. Anal. 2014, 17, 1158-1174.

25. Dabas, J.; Chauhan, A. Existence and uniqueness of mild solution for an impulsive neutral fractional integro-differential equation with infinite delay. Math. Comput. Modelling. 2013, 57, 754-763.

26. Hale, J.; Kato, J. Phase space for retarded equations with infinite delay. Funkcial. Ekvac. 1978, 21, 11-41.

27. Agarwal, R.P.; Benchohra, M.; Hamani, S. A survey on existence results for boundary value problems of nonlinear fractional differential equations and inclusions. Acta. Appl. Math. 2010, 109, 973-1033.

28. Maleknejad, K.; Mollapourasl, R.; Nouri, K. Study on existence of solutions for some nonlinear functional-integral equations. Nonlinear. Anal. 2008, 69, 2582-2588.

29. Smart, D.R. Fixed Point Theorems. Cambridge University Press: Cambridge, 1980. 